\title{
SynV and SynX: A story more than DNA synthesis
}

\author{
Hengqian $\operatorname{Ren}^{1}$ \& Huimin Zhao ${ }^{1,2,3^{*}}$ \\ ${ }^{1}$ Department of Chemical and Biomolecular Engineering, University of Illinois at Urbana-Champaign, Urbana, IL 61801, USA; \\ ${ }^{2}$ Department of Chemistry and Biochemistry, Bioengineering, University of Illinois at Urbana-Champaign, Urbana, IL 61801, USA; \\ ${ }^{3}$ Carl R. Woese Institute for Genomic Biology, University of Illinois at Urbana-Champaign, Urbana, IL 61801, USA
}

Received March 29, 2017; accepted April 1, 2017; published online May 5, 2017

Citation: $\quad$ Ren, H., and Zhao, H. (2017). SynV and SynX: A story more than DNA synthesis. Sci China Life Sci 60, 558-560. doi: 10.1007/s11427-017-9039-6

Genomes have been studied by biologists for decades with a goal to decipher the origin, evolution, and nature of life on earth. These studies often rely on genetic manipulations such as deletions and insertions (a top-down approach), but recent advances in DNA synthesis provide a new optionwhole genome synthesis (a bottom-up approach). While several bacterial and viral genomes have been successfully synthesized, the yeast genome synthesis project (Sc2.0) aims to synthesize the first eukaryotic genome-all 16 chromosomes of Saccharomyces cerevisiae (Kosuri and Church, 2014). Due to its complexity and large scale, this project has been carried out by an international consortium that consists of approximately two hundred researchers from over 10 universities and institutions from five countries. Following the construction of the first yeast chromosome synIII in 2014 (Annaluru et al., 2014), this international consortium recently achieved another milestone by completing the synthesis of five yeast chromosomes.

As a key player in the Sc2.0 international consortium, Yuan and coworkers from the Tianjin University (TJU) completed the synthesis of redesigned yeast chromosomes synV and synX (Wu et al., 2017; Xie et al., 2017). Hundreds of building blocks, the $\sim 750$ bp primary DNA segments and 2 to $4 \mathrm{~kb}$ mini chunks, were built from oligonucleotides and used to replace the corresponding DNA segments in the native chromosomes stepwisely, resulting in two synthetic chromosomes synV and synX. In addition to the enormous cloning effort for building more than $1.2 \mathrm{Mb}$ DNA from scratch, fixing the unwanted sequences in the

*Corresponding author (email: zhao5@illinois.edu) synthetic chromosomes is another major obstacle. By using the integrative cotransformation method and a clustered regularly interspaced short palindromic repeats (CRISPR)/ CRISPR-associated protein 9 (Cas9)-based genome editing method, all 3331 bp randomly distributed mutations and other unpredictable events were corrected in the initial synV isolate in order to perfectly match the designer sequence. While in the synthesis of synX, meiotic crossover was employed to fix the massive rearrangements and duplications.

Apart from the synthesis of synV and synX, Yuan and coworkers developed new tools for whole genome synthesis and analysis. For example, they developed a method called pooled PCR Tag mapping (PoPM) for mapping the bugs, i.e. genetic alterations that affect cell fitness, in the designer chromosomes. By using the Sc2.0 PCR tag system and pooling strategy, PoPM may be generalized to assess any watermarked chromosome, which can serve as a powerful debugging method for building designer chromosomes in the future. Moreover, they created a circularized synV and compared its phenotype with that of the linear synV in $S$. cerevisiae. Such comparative study not only extends genome design principles, but also provides a model for studying genomic rearrangement, ring chromosome evolution, and human ring chromosome disorders.

It is worth to be mentioned that along with the scientific and technological achievements, Yuan and coworkers developed a mechanism to educate the next-generation synthetic biologists. Sixty-one undergraduate students from the course "Build-A-Genome (BAG) China" in the TJU were engaged in the synthesis of chromosome V. Although most of them had no or little prior experience in DNA synthesis, 
through BAG training, they not only learned knowledge and techniques for DNA synthesis, but also gained first-hand experience as researchers such as performing troubleshooting which can hardly be provided by regular courses. After the BAG training, some of these undergraduate students decided to continue their education in synthetic biology by enrolling either the master or Ph.D. programs. Furthermore, by getting involved in an international research team where all the expertise and resources are shared with no obstacles, students can get a direct impression of the importance of international collaboration.

The contribution made by Yuan and coworkers to the Sc 2.0 project is far beyond just constructing the synthetic chromosomes synV and synX. The knowledge generated from the characterization and debugging of synthetic chromosomes is also extremely invaluable. Even though the Sc2.0 project currently desires to maintain the "wild-type" phenotype of $S$. cerevisiae and hence its design principles are very conservative, the arbitrary modifications at a systematic level could inevitably lead to unexpected outcomes. Thus, as part of the goal of the Sc 2.0 project, through genome synthesis, researchers may be able to address many biological questions that are difficult to approach in the past. By mapping the phenotypes caused by the designer sequences stepwisely, Yuan and coworkers did an excellent job in the "design-build-test-learn" cycle. Their efforts not only expanded our understanding of the yeast genome, but also extended the design principles of synthetic genomes, which can guide the engineering of yeast as well as other organisms with novel functionalities. On the other hand, due to the highly diverse expertise in the consortium, Yuan and coworkers, as well as other participating research groups, benefited from the global collaboration. For example, the characterization of 3D organization of synV and synX was carried out by Muller's group and Koszul's group (Mercy et al., 2017). Some of the researchers involved also benefited from the international consortium by getting a chance to work overseas.

The synthesis of SynV and synX, together with the other three yeast chromosomes, undoubtedly marks an important milestone in the Sc2.0 project. However, more improvements and discoveries are expected in the near future. First, although the synthesis of all 16 chromosomes of $S$. cerevisiae could be finished by the end of 2017 as claimed by the Sc2.0 team, the synthetic strain is only expected to exhibit similar performance as the wild type due to the conservative design. Synthetic biologists are keen to see artificial yeasts with more intriguing designer properties, as well as their corresponding industrial applications such as fermentation and disease treatment. From a scientific point of view, biological questions, such as how transposons evolve and spread throughout a host genome, are also awaiting to be solved. All these goals require the $\mathrm{Sc} 2.0$ team to develop more aggressive design principles and start new "de- sign-build-test-learn" cycles, and to perform more in-depth study of the yeast with artificial chromosomes. Second, currently the construction of designer chromosomes is still the rate-limiting step in the Sc2.0 project. The workflow for chromosome synthesis is relatively tedious and timeconsuming. Due to its low throughput, this workflow can hardly match the ever-increasing need for building designer yeast and other organisms. In addition to develop faster and more efficient DNA synthesis and assembly methods, incorporation of automation technologies into the workflow is essential. Third, the high cost for DNA synthesis makes high-throughput genome synthesis cost prohibitive. Thus, novel cheaper DNA synthesis technologies are highly desirable. Overall, the recent achievements by the $\mathrm{Sc} 2.0$ international consortium have moved a step closer to the creation of the first eukaryotic organism with a fully chemically synthesized genome, and paved the way for other genome synthesis projects such as the synthesis of the human genome.

Compliance and ethics The author(s) declare that they have no conflict of interest.

Annaluru, N., Muller, H., Mitchell, L.A., Ramalingam, S., Stracquadanio, G., Richardson, S.M., Dymond, J.S., Kuang, Z., Scheifele, L.Z., Cooper, E.M., Cai, Y.Z., Zeller, K., Agmon, N., Han, J.S., Hadjithomas, M., Tullman, J., Caravelli, K., Cirelli, K., Guo, Z.Y., London, V., Yeluru, A., Murugan, S., Kandavelou, K., Agier, N., Fischer, G., Yang, K., Martin, J.A., Bilgel, M., Bohutski, P., Boulier, K.M., Capaldo, B.J., Chang, J., Charoen, K., Choi, W.J., Deng, P., DiCarlo, J.E., Doong, J., Dunn, J., Feinberg, J.I., Fernandez, C., Floria, C.E., Gladowski, D., Hadidi, P., Ishizuka, I., Jabbari, J., Lau, C.Y.L., Lee, P.A., Li, S., Lin, D., Linder, M.E., Ling, J., Liu, J., Liu, J., London, M., Ma, H., Mao, J., McDade, J.E., McMillan, A., Moore, A.M., Oh, W.C., Ouyang, Y., Patel, R., Paul, M., Paulsen, L.C., Qiu, J., Rhee, A., Rubashkin, M.G., Soh, I.Y., Sotuyo, N.E., Srinivas, V., Suarez, A., Wong, A., Wong, R., Xie, W.R., Xu, Y.J., Yu, A.T., Koszul, R., Bader, J.S., Boeke, J.D., and Chandrasegaran, S. (2014). Total Synthesis of a Functional Designer Eukaryotic Chromosome. Science 344, 55-58.

Kosuri, S., and Church, G.M. (2014). Large-Scale de novo DNA Synthesis: Technologies and Applications. Nat Methods 11, 499-507.

Mercy, G., Mozziconacci, J., Scolari, V.F., Yang, K., Zhao, G., Thierry, A., Luo, Y., Mitchell, L.A., Shen, M., Shen, Y., Walker, R., Zhang, W., Wu, Y., Xie, Z.X., Luo, Z., Cai, Y., Dai, J., Yang, H., Yuan, Y.J., Boeke, J.D., Bader, J.S., Muller, H., and Koszul, R. (2017). 3D Organization of Synthetic and Scrambled Chromosomes. Science 355, eaaf4597.

Wu, Y., Li, B.Z., Zhao, M., Mitchell, L.A., Xie, Z.X., Lin, Q.H., Wang, X., Xiao, W.H., Wang, Y., Zhou, X., Liu, H., Li, X., Ding, M.Z., Liu, D., Zhang, L., Liu, B.L., Wu, X.L., Li, F.F., Dong, X.T., Jia, B., Zhang, W.Z., Jiang, G.Z., Liu, Y., Bai, X., Song, T.Q., Chen, Y., Zhou, S.J., Zhu, R.Y., Gao, F., Kuang, Z., Wang, X., Shen, M., Yang, K., Stracquadanio, G., Richardson, S.M., Lin, Y., Wang, L., Walker, R., Luo, Y., Ma, P.S., Yang, H., Cai, Y., Dai, J., Bader, J.S., Boeke, J.D., and Yuan, Y.J. (2017). Bug Mapping and Fitness Testing of Chemically Synthesized Chromosome X. Science 355, eaaf4706.

Xie, Z.X., Li, B.Z., Mitchell, L.A., Wu, Y., Qi, X., Jin, Z., Jia, B., Wang, X., Zeng, B.X., Liu, H.M., Wu, X.L., Feng, Q., Zhang, W.Z., Liu, W., Ding, M.Z., Li, X., Zhao, G.R., Qiao, J.J., Cheng, J.S., Zhao, M., Kuang, Z., Wang, X., Martin, J.A., Stracquadanio, G., Yang, K., Bai, 
X., Zhao, J., Hu, M.L., Lin, Q.H., Zhang, W.Q., Shen, M.H., Chen, S., Su, W., Wang, E.X., Guo, R., Zhai, F., Guo, X.J., Du, H.X., Zhu, J.Q., Song, T.Q., Dai, J.J., Li, F.F., Jiang, G.Z., Han, S.L., Liu, S.Y., Yu, Z.C., Yang, X.N., Chen, K., Hu, C., Li, D.S., Jia, N., Liu, Y., Wang, L.T., Wang, S., Wei, X.T., Fu, M.Q., Qu, L.M., Xin, S.Y., Liu, T., Tian, K.R., Li, X.N., Zhang, J.H., Song, L.X., Liu, J.G., Lv, J.F., Xu,
H., Tao, R., Wang, Y., Zhang, T.T., Deng, Y.X., Wang, Y.R., Li, T., Ye, G.X., Xu, X.R., Xia, Z.B., Zhang, W., Yang, S.L., Liu, Y.L., Ding, W.Q., Liu, Z.N., Zhu, J.Q., Liu, N.Z., Walker, R., Luo, Y., Wang, Y., Shen, Y., Yang, H., Cai, Y., Ma, P.S., Zhang, C.T., Bader, J.S., Boeke, J.D., and Yuan, Y.J. (2017). "Perfect" designer chromosome V and behavior of a ring derivative. Science 355 , eaaf 4704 